\title{
Cycle Length Dependence of Human Action Potential Duration In Vivo Effects of Single Extrastimuli, Sudden Sustained Rate Acceleration and Deceleration, and Different Steady-State Frequencies
}

\author{
Michael R. Franz, Charles D. Swerdlow, L. Bing Liem, and Jochen Schaefer \\ Divisions of Cardiology, Medical School of the Christian Albrechts University, D-2300 Kiel, German Federal Republic, \\ and Stanford University, Stanford, California 94305
}

\begin{abstract}
Using a new method for long-term recording of monophasic action potentials from the human heart, we studied in 17 patients the effects on ventricular action potential duration (APD) of three clinically pertinent cycle length perturbations: (1) single extrastimuli, (2) abrupt sustained rate acceleration and deceleration, and (3) different steady-state cycle lengths. Results were: (a) APD after single extrastimuli at progressively longer cycle lengths were related to the extrastimulus cycle length with a biphasic electrical restitution curve which after an initial steep rise and a subsequent transient descent rose again more gradually to a plateau at cycle lengths above 800-1,000 ms. (b) After a sustained step decrease in cycle length, the first APD shortened abruptly while final steadystate adaptation required up to several minutes. The transition between the rapid and slow phase of APD change was characterized by a variable alternans of APD which correlated inversely with the preceding diastolic interval. (c) In the steady state, APD correlated linearly with cycle length, increasing an average of $23 \mathrm{~ms}$ per $100 \mathrm{~ms}$ cycle length increase $(r=0.995)$. The divergence between steady-state and non-steady-state APD, and the slowness of steady-state adaptation, are important factors to be considered in clinical electrophysiologic studies and in rate correction algorithms of APD or QT intervals, respectively.
\end{abstract}

\section{Introduction}

Changes in the duration of the cardiac action potential resulting from changes in cycle length have been investigated extensively in isolated tissue preparations (1-13), but only limited data exist about the cycle length dependence of the human ventricular action potential duration (APD) ${ }^{1}$ in situ. Because substantial differences in cycle length dependence exist among species (2) and because removal of myocardial tissue from its natural environment may alter electrophysiologic properties, it is desirable to establish the cycle length dependence of the human APD in vivo. Inferences from $Q-T$ interval measure-

Address reprint requests to Dr. Franz, Cardiac Arrhythmia Unit, Cardiology Division, CVRC 293, Stanford University School of Medicine, 300 Pasteur Drive, Stanford, CA 94305.

Received for publication 3 April 1987 and in revised form 26 April 1988.

1. Abbreviation used in this paper: APD, action potential duration.

J. Clin. Invest.

(c) The American Society for Clinical Investigation, Inc.

0021-9738/88/09/0972/08 \$2.00

Volume 82, September 1988, 972-979 ments of the electrocardiogram $(7,13)$ are of limited value because the correlation between this summated signal and the APD, which varies from site to site in the ventricle (14-16), is unclear. Similarly, refractory period determinations (17-19) are only indirect measures of repolarization and are impractical for assessing APD changes over many consecutive beats. Some direct APD measurements have been obtained from the human heart using suction electrodes (20). However, suction electrodes must be withdrawn from human myocardium within less than 2 min to avoid injury (21) and therefore cannot be used to measure longer-term changes in APD, such as those over several basic cycle lengths.

We have developed a new contact electrode catheter technique which permits safe long-term monophasic action potential recording from a single endocardial site of the human heart (22). Our goals were to establish for the in situ human ventricle the dependence of APD on three clinically pertinent cycle length alterations: $(a)$ single cycle length perturbations introduced during steady-state pacing, $(b)$ abrupt sustained increments and decrements in cycle length, and $(c)$ different steady-state cycle lengths.

\section{Methods}

Patients. Studies were carried out in 17 patients undergoing clinically indicated electrophysiologic investigation. All patients gave written, informed consent to the study protocol which had been approved by the institutions' committee on human investigation. None of the patients received any antiarrhythmic drugs, including digoxin and beta blockers, for a period of at least five half-lives prior to study. Indications for invasive electrophysiologic or hemodynamic study were suspected coronary artery disease in seven patients, supraventricular tachycardia in two, Wolff-Parkinson-White syndrome in two, sicksinus-syndrome in three, sustained ventricular tachycardia in one, and nonsustained ventricular tachycardia in two. All patients had normal electrocardiograms and QT durations. Eight patients had intrinsic heart rates that permitted exploration of a wide range of pacing rates without escape beats. No patient had angina or presented electrocardiographic signs of ischemia during the periods of rapid pacing.

Monophasic action potentials were recorded with a contact electrode catheter as previously described (22). Recordings from this electrode catheter have been shown to accurately indicate the onset, duration, configuration, and relative changes in amplitude of transmembrane action potentials (23). The catheter was placed with its tip electrode against the endocardium of the right ventricular apex in 15 patients and the right ventricular outflow tract in two patients. Monophasic action potentials were recorded continuously from the same site as judged by fluoroscopic control and, more importantly, by smooth uninterrupted waveforms during the entire recording period. Recordings with even subtle baseline shifts were excluded from analysis. The seven patients with suspected coronary artery disease had electrophysiologic measurements begun at least $30 \mathrm{~min}$ after intracoronary dye injection, an interval long enough for dye-related electrophysiologic changes to subside (24). 
A pacing catheter was introduced into the right atrium (three patients) or right ventricle (14 patients) and pacing stimuli of twice diastolic threshold were delivered by a custom-built programmable stimulator to investigate the following three aspects of cycle-length dependence of human APD.

Electrical restitution. The time course with which APD recovers as a function of the interval between a steady-state response and a subsequent test response, introduced at progressively longer test intervals, is known as the electrical restitution curve (25). Hearts were paced at a constant basic cycle length until APD reached a steady state. A single extrastimulus was then applied $10 \mathrm{~ms}$ after the effective refractory period. Subsequent extrastimuli were introduced with $10 \mathrm{~ms}$ increments up to a cycle length of $400 \mathrm{~ms}$ and then with 20 - or 50 -ms increments until escape beats occurred. After each extrastimulus, regular pacing at the basic cycle length was resumed until recovery of the original steady state (usually within $10-20$ beats). Electrical restitution curves were determined in seven patients at a basic cycle length of 600 $\mathrm{ms}$ and in two patients at a basic cycle length of 700 and $430 \mathrm{~ms}$.

Rate adaptation. To examine the time course of change in APD after a sudden, sustained change in cycle length, the hearts were first paced at a constant basic cycle length of 500-1,000 ms until APD had reached a steady state. The basic cycle length was then abruptly decreased by at least $250 \mathrm{~ms}$ (see Table I for individual cycle length changes) and maintained constant at the new cycle length until APD had reached its new steady state. Each step change in cycle length was accomplished from one stimulus to the next. This protocol was successfully completed in 10 patients. Reversal of APD adaptation was studied in five patients by switching back to the original longer basic cycle length and continuing recording until APD had returned to baseline. In one patient, the effect on APD of two consecutive increases and decreases in basic cycle length could be monitored continuously on a beat-to-beat basis while the recording catheter remained in a stable position.

Steady-state relation. Pacing was performed at a constant basic cycle length just below the spontaneous cycle length until APD had reached a steady state. The paced cycle length was then decreased in decrements of $50-100 \mathrm{~ms}$ up to a minimum of $300-400 \mathrm{~ms}$, each step being maintained for $3 \mathrm{~min}$ to assure steady state. It was possible to combine this protocol with the one on electrical restitution in two patients and with the one on rate adaptation in another two with the recording catheter kept at the same endocardial site.

Data analysis. Monophasic action potentials and three to six surface ECG leads were displayed simultaneously on a multichannel recorder and stored on magnetic tape. Data were analyzed manually from off-line recordings at a paper speed of $250 \mathrm{~mm} / \mathrm{s}$ or by computer after recordings were digitized at a sampling rate of $1 \mathrm{kHz}$ (23): Pacing trains which were interrupted by unpaced premature beats or supraventricular or ventricular tachycardia were excluded from analysis. APD was measured as the time from rapid depolarization to $90 \%$ repolarization. The interval between depolarization phases of two consecutive action potentials, rather than the stimulus interval, was defined as the cycle length in order to exclude influences of conduction time. The interval from $90 \%$ repolarization to the upstroke of the subsequent APD was defined as the electrical diastolic interval. Steady-state APD was the average of eight consecutive complexes at each basic cycle length, recorded continuously at a single endocardial site. To resolve even small changes in APD during cycle length adaptation, recordings of the last 10 beats preceding and the first 50 beats after the cycle length change were analyzed on a beat-to-beat basis and plotted as individual data points. Linear regression analysis was used to assess the correlation between steady-state APD and cycle length.

\section{Results}

At a single site and during constant steady-state pacing, the duration and configuration of monophasic action potential recordings was stable within $5 \mathrm{~ms}$ or $2 \%$ for test periods of $1 \mathrm{~h}$.
This high stability of the steady-state APD was a prerequisite for interpreting some of the more subtle APD changes described below.

Electrical restitution curve. The effect of single cycle length perturbations during steady state pacing is shown in Fig. 1. The earliest action potential that could be elicited by an extrastimulus occurred at $\sim 70 \%$ repolarization of the preceding steady-state response. The duration of this most premature action potential was considerably less than the steady-state APD. With progressively increasing extrastimulus cycle lengths, APD first rose steeply to an early maximum at 50-150 $\mathrm{ms}$ after the effective refractory period of the steady-state response, decreased again with longer, but still less than steadystate, cycle length, and then gradually increased to a maximum value at $\sim 1,000 \mathrm{~ms}$ of cycle length. The early biphasic "hump" of the electrical restitution curves was due to so-called "supernormal" (2) premature action potentials which have a greater plateau duration and slope of phase 3 than those elicited at slightly shorter or longer extrastimulus intervals or the steady-state responses.

Abrupt sustained cycle length changes. Fig. 2 shows original action potential recordings during a step decrease and a subsequent step increase in cycle length. APD did not adjust immediately to the new cycle length but required more than 2 min or several hundred beats to reach a new steady state. Conversely, when at the new steady state the original, long cycle length was abruptly resumed, APD remained shorter than before the period of rapid pacing and only gradually adjusted to the longer cycle length.

Fig. 3 quantitatively depicts the time course of action potential shortening and lengthening after two sequential step decreases and increases in paced cycle length. The first action potential after the step decrease in cycle length shows abrupt shortening; thereafter, action potential shortening occurs with a much slower time course until steady state is reached. Conversely, when the paced cycle length is increased abruptly to the baseline value, the first action potential at the longer cycle length shows abrupt prolongation, followed by a much slower time course of lengthening.

Table I lists APD adaptation times after 10 sudden rate accelerations in nine patients. Adaptation times ranged from 1.8 to $3.2 \mathrm{~min}$, with more than hundred beats required to achieve $75 \%$ of the steady-state level. There was only weak correlation between the magnitude of absolute $(r=0.29)$ or

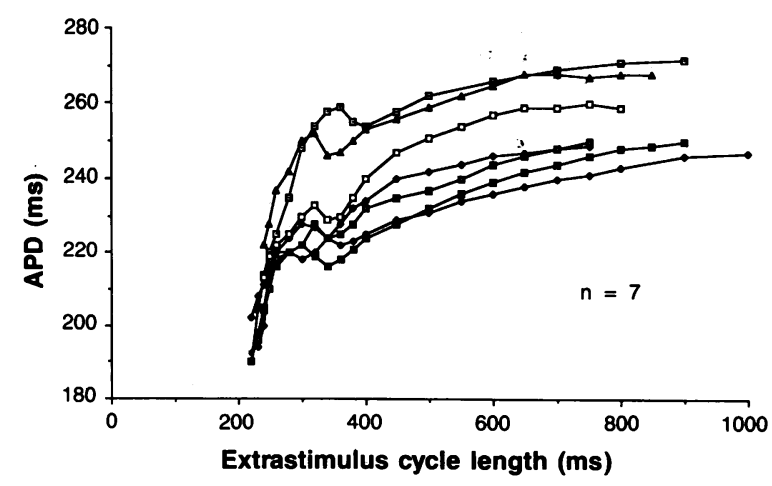

Figure 1. Electrical restitution curves of right vehtricular myocardium at a basic drive cycle length of $600 \mathrm{~ms}(n=7)$. Note the biphasic time course of the early part of electrical restitution. 


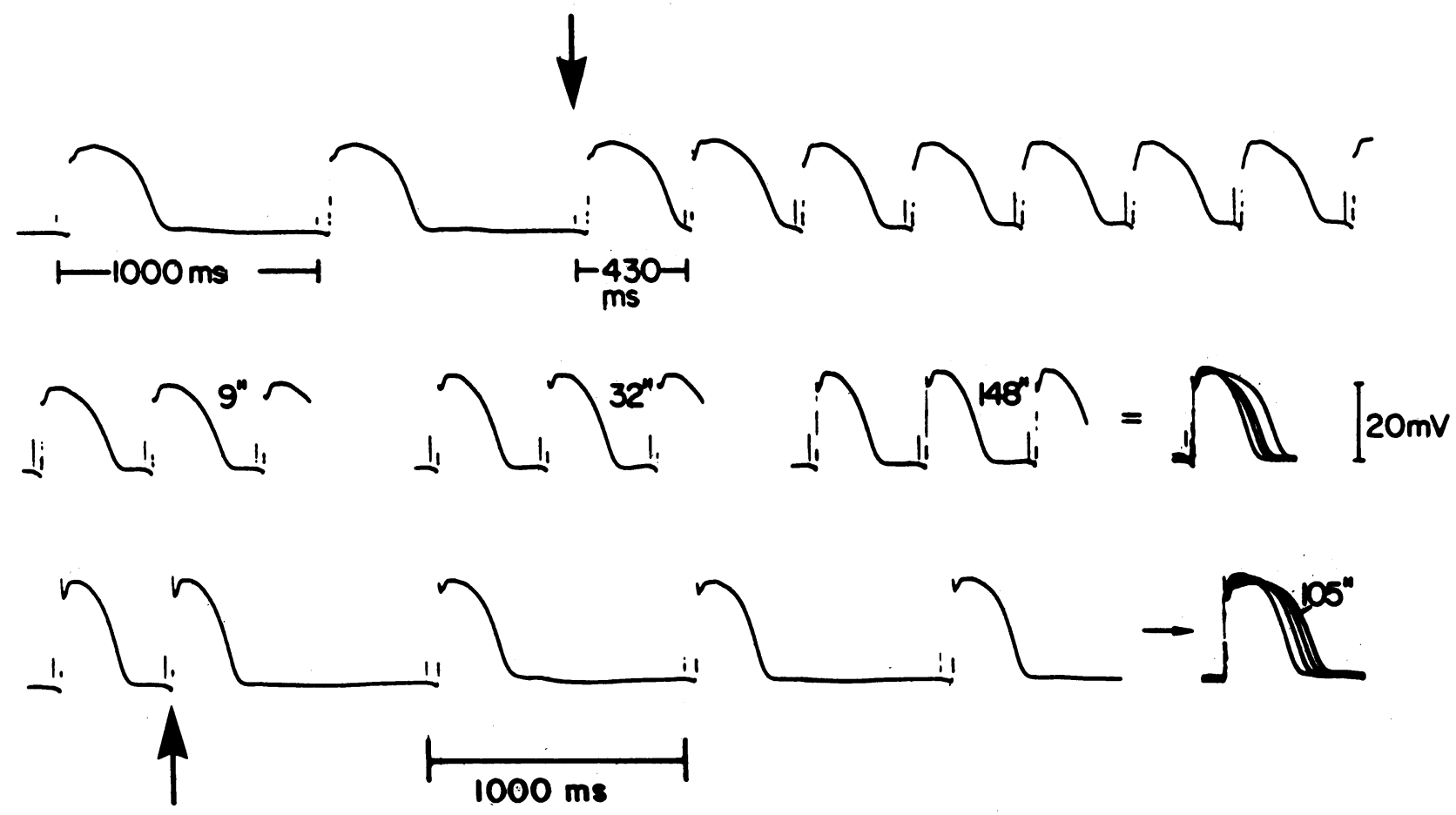

Figure 2. Original action potentials recorded continuously from a single right ventricular site during a sequential step decrease and increase in cycle length (patient 2). Upper trace: After 3 min of pacing at a basic cycle length of $1,000 \mathrm{~ms}$ (steady-state APD $=330 \mathrm{~ms}$ ), the cycle length was suddenly decreased to $430 \mathrm{~ms}$. Note progressive shortening of action potentials after rate increase (first six beats are shown). Middle trace: APD continued to shorten slowly as shown by the examples at indicated time intervals. Because after the step decrease in cycle length the cycle length was kept constant, APD short-

ening was associated with reciprocal lengthening of the duration of the electrical diastolic interval. Lower trace: When after 3 min of constant pacing at the shorter cycle length APD had reached a new steady state ( $=210 \mathrm{~ms}$ ), the cycle length was switched back to 1,000 ms. APD gradually increased and after several hundred beats, recovered to the initial steady-state duration. The superimposed recordings demonstrate that these slow changes in APD were mainly due to a change in plateau duration.

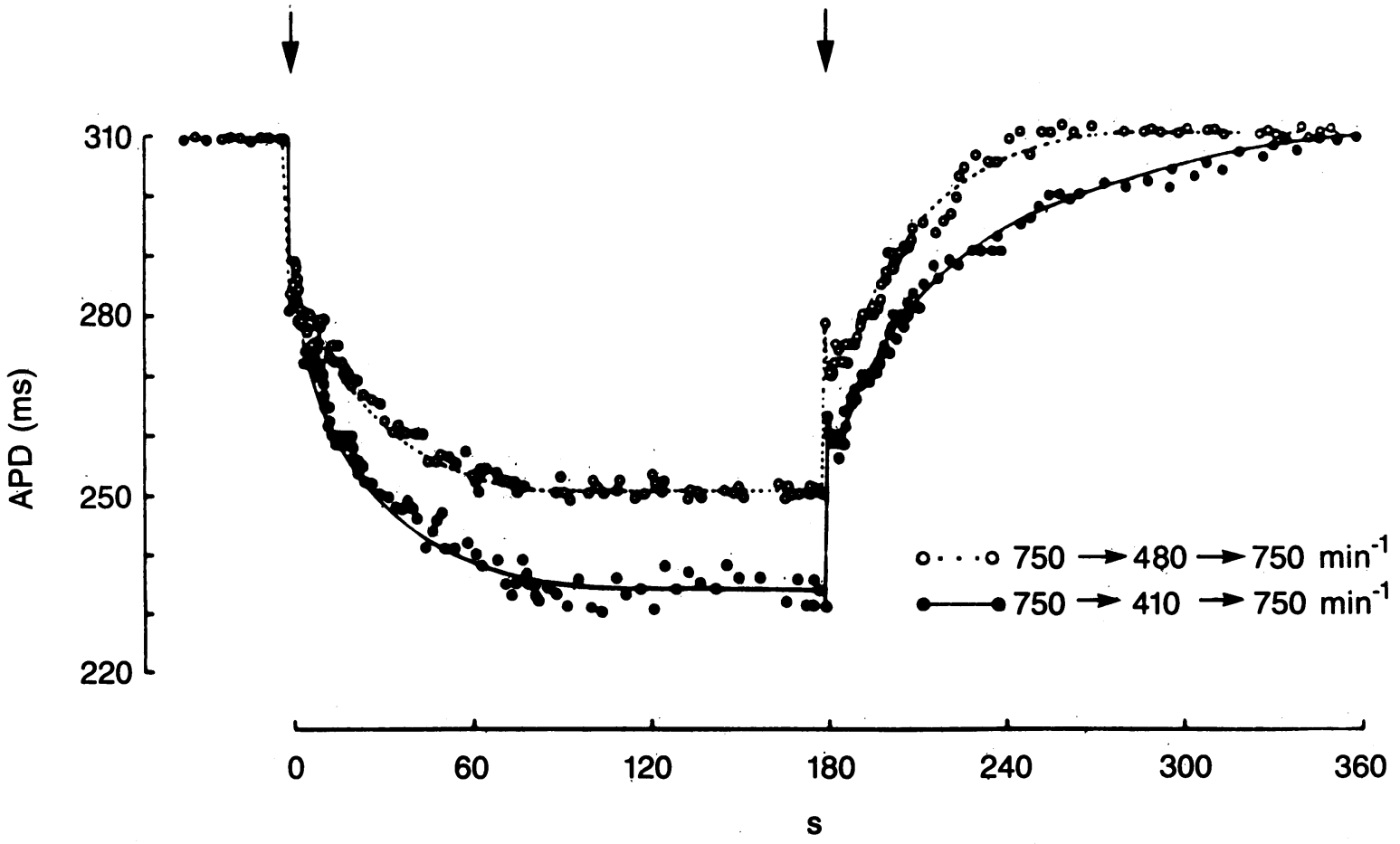

Figure 3. Time course of APD adaptation after two consecutive step decreases and increases in cycle length (patient 8). Both the step increases and decreases in pacing rate are followed by an initial rapid phase and a subsequent slow phase of action potential shortening and lengthening, respectively. The rapid phase of APD change showed "notching," which is demonstrated in more detail in Fig. 4. 
Table I. APD Shortening at Selected Points during Steady-State Adaptation after Sudden Sustained Decrements in Cycle Length

\begin{tabular}{|c|c|c|c|c|c|c|c|}
\hline \multirow[t]{2}{*}{ Patient } & \multirow[t]{2}{*}{$\Delta \mathrm{CL}$} & \multicolumn{2}{|c|}{$\Delta$ 1st AP } & \multicolumn{2}{|c|}{$\Delta$ 8th AP } & \multirow{2}{*}{$\frac{\Delta \mathrm{AP} \text { at ss }}{m s}$} & \multirow{2}{*}{$\frac{\text { Time to ss }}{\min }$} \\
\hline & & $m s$ & $\%$ & $m s$ & $\%$ & & \\
\hline 1 & $800-400$ & 8 & 8.9 & 24 & 26.7 & 90 & 3.2 \\
\hline 2 & $1,000-430$ & 20 & 16.7 & 50 & 41.7 & 120 & 2.5 \\
\hline 3 & $500-300$ & 12 & 21.4 & 24 & 42.9 & 56 & 2.5 \\
\hline 4 & $600-350$ & 10 & 22.7 & 18 & 40.9 & 44 & 3.0 \\
\hline 5 & $630-330$ & 23 & 35.4 & 35 & 53.8 & 65 & 1.8 \\
\hline 6 & $800-400$ & 15 & 18.8 & 24 & 30.0 & 80 & 3.0 \\
\hline 7 & $650-400$ & 10 & 20.0 & 21 & 42.0 & 50 & 2.5 \\
\hline 8 & $750-410$ & 30 & 37.5 & 38 & 47.5 & 80 & 3.0 \\
\hline 9 & $750-480$ & 27 & 45.0 & 35 & 58.3 & 60 & 2.0 \\
\hline 10 & $600-380$ & 11 & 25.0 & 21 & 47.7 & 44 & 2.2 \\
\hline Mean \pm SD & & & $25.1 \pm 10.4$ & & $43.2 \pm 9.1$ & & $2.6 \pm 0.5$ \\
\hline
\end{tabular}

The amount of shortening of the 1st, 8th, and 20th response (in percentage of final steady-state adaptation), the amount of shortening reached at steady state (in milliseconds), and the time required to reach steady state are shown. $\Delta \mathrm{CL}$, decrements in cycle length; ss, steady state.
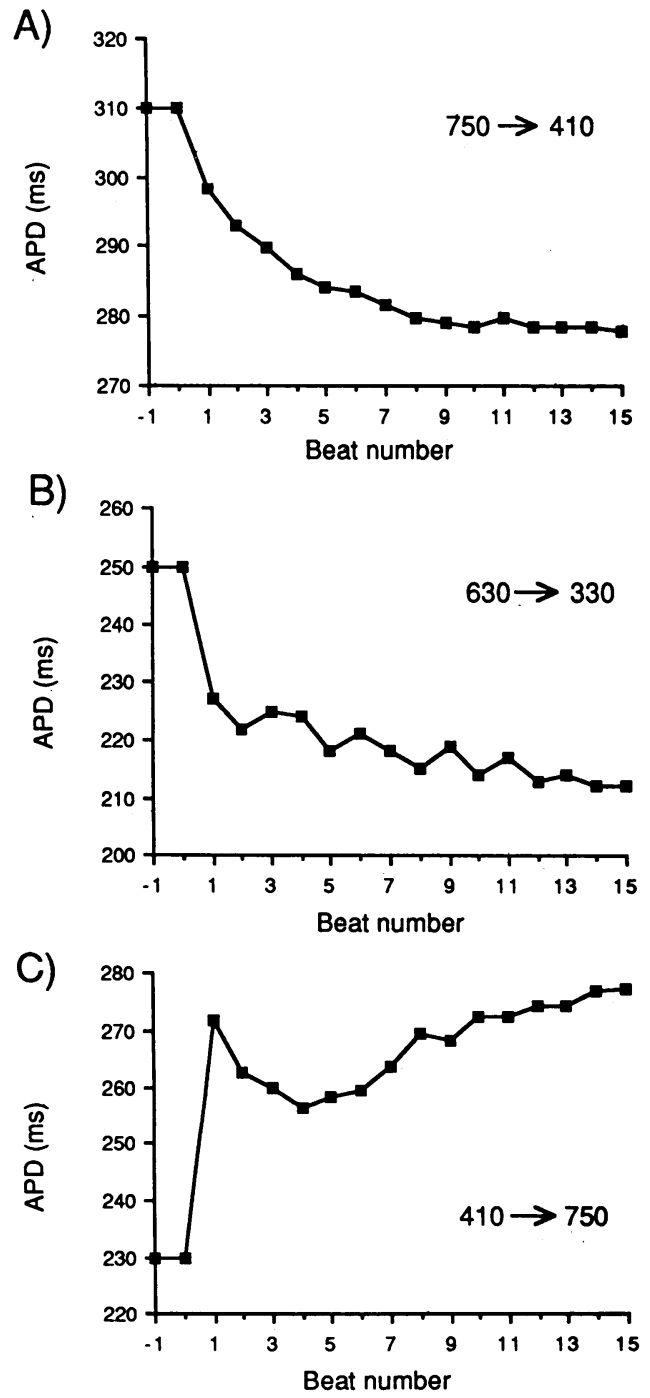

Figure 4. Examples of APD changes during the first 15 beats after a step decrease or increase in cycle length. percent $(r=0.35)$ change from the initial cycle length and the time required for steady state adaptation. Table I also indicates the relative contributions of the first and eighth response after the cycle length decrease to the final steady-state shortening; the eighth response, usually considered steady state in clinical electrophysiologic studies, represents only $43.2 \pm 9.1 \%$ of the change towards steady state.

Figs. 4 and 5 detail the changes in APD that occurred during the first 15 beats after a step change in cycle length. The first APD after a step decrease in cycle length was always shorter than the preceding steady-state APD. Subsequent shortening occurred either in continuous, decremental fashion (Fig. $4 A$ ) or with an irregular pattern where shortening over several beats was interrupted by one or several responses with longer or constant APD (Fig. 4 B). After a step increase in cycle

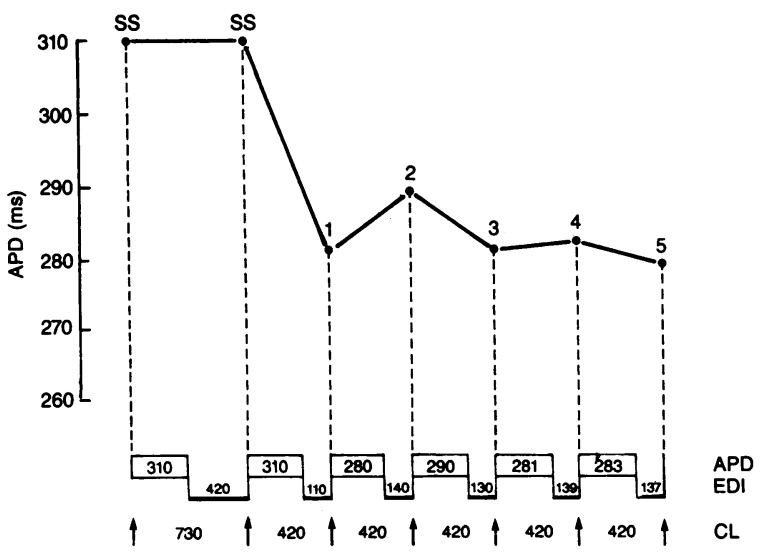

Figure 5. APD changes during the first 5 beats after a sudden decrease in cycle length $(C L)$ from 730 to $420 \mathrm{~ms}$ are shown together with changes in electrical diastolic intervals $(E D I)$. Because after the step change in cycle length, cycle length was kept constant, each change in APD resulted in a reciprocal change in EDI preceding the subsequent action potential. A longer APD produced a shorter EDI, which in turn was followed by a shorter APD and a subsequently longer EDI. 
length, the first APD was always longer than the preceding steady-state response, while the subsequent two to five responses shortened again (despite the longer and constant cycle length), before gradual lengthening proceeded towards the new steady state (Fig. $4 C$ ).

APD is a function of the duration of electrical diastole, not the total cycle length (9). Because the cycle length was kept constant after each step change, each change in APD must result in an identical, but reciprocal change in diastole. We therefore compared APD changes with changes in the duration of electrical diastole preceding each of the first several beats after a rate change (Fig. 5). Relative shortening of APD was greatest for the first beat at the shorter cycle length, and thus was relative lengthening of subsequent diastole. This was followed by an increase in the next APD and a decrease in the next diastolic interval. This cycle repeated itself in a dampened pattern.

Steady-state relationship. Fig. 6 depicts the steady-state relationship between cycle length and APD for seven patients in whom action potential recording at a single site could be maintained long enough to allow for steady-state pacing over a large range of cycle lengths, with each cycle length kept constant for $3 \mathrm{~min}$. In each patient, APD increased linearly with basic cycle length from 350 to $\sim 800 \mathrm{~ms}$. Over this range, APD increased an average of $23 \mathrm{~ms}$ for each 100 -ms increment in basic cycle length (Table II). At very long steady-state cycle length (above 800-1,000 ms), APD deviated from the linear relation and approached a plateau value. This is particularly apparent in the one patient in whom regular pacing at cycle lengths as long as $1,300 \mathrm{~ms}$ could be maintained without escape beats. As shown by representative original recordings (Fig. 6, inset), changes in steady-state APD were due to a change in action potential plateau duration while the slope of final repolarization remained unaltered.

Nonsteady-state vs. steady-state APD. Fig. 7 compares the APD following single premature or postmature stimuli with

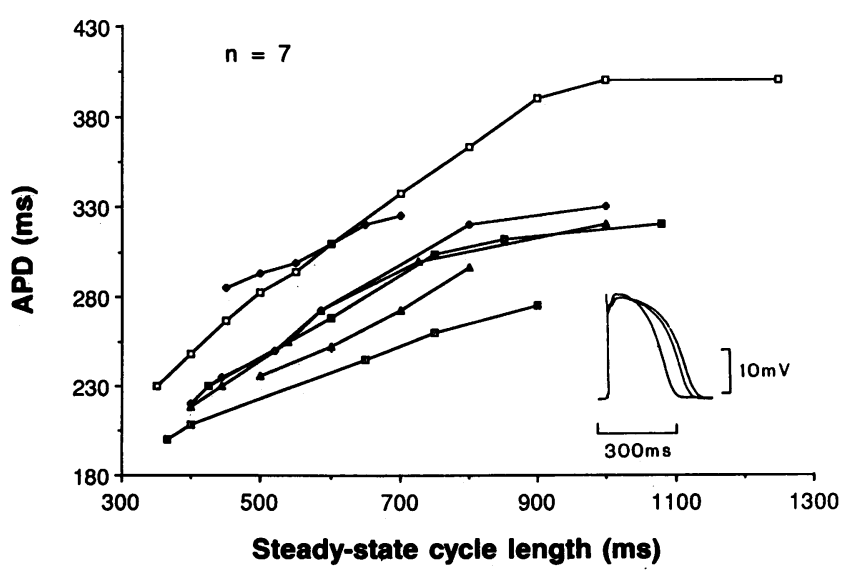

Figure 6. APD as a function of cycle length in the steady state. Data points represent the average duration of eight consecutive responses at the end of a 3-min train of regular pacing. Because variance of APD at each cycle length was $<2 \%$, standard deviation bars are not shown. (Inset) Accompanying configurational changes of the mean action potential. Linear regression parameters of these data (except those at very long cycle lengths where APD deviated sharply from the linear relation) are presented in Table II.
Table II. Linear Regression Analysis of the Relation between APD and Basic Cycle Length in the Steady State

\begin{tabular}{cccc}
\hline Patient & BCL range & Slope & $r$ \\
\hline & $m s$ & & \\
2 & $350-850$ & 0.147 & 0.997 \\
3 & $365-720$ & 0.239 & 0.988 \\
4 & $400-725$ & 0.227 & 0.998 \\
8 & $300-850$ & 0.300 & 0.996 \\
9 & $445-650$ & 0.190 & 0.993 \\
11 & $365-750$ & 0.261 & 0.997 \\
12 & $490-750$ & 0.194 & 0.983 \\
Mean \pm SD & & $0.229 \pm 0.049$ & $0.995 \pm 0.003$
\end{tabular}

All data presented graphically in Fig. 7 were used, except those at very long cycle length where APD deviated clearly from linearity. $\mathrm{BCL}$, basic cycle length.

the steady-state APD at corresponding cycle lengths. APD of steady-state beats differed considerably from those that immediately followed a cycle length change, and the difference progressively increased as the difference in cycle length increased.

\section{Discussion}

Because APD is known to vary substantially from site to site in the human ventricle (16), it was important to monitor cycle length-related APD changes at the same ventricular site. The contact electrode technique used in this study was amenable to long-term recording of monophasic action potentials from human endocardium; it provided steady-state APD with a variance of $<2 \%$ and often allowed us to study the effect of more than one pacing protocol on local repolarization properties while stability of recordings was maintained.

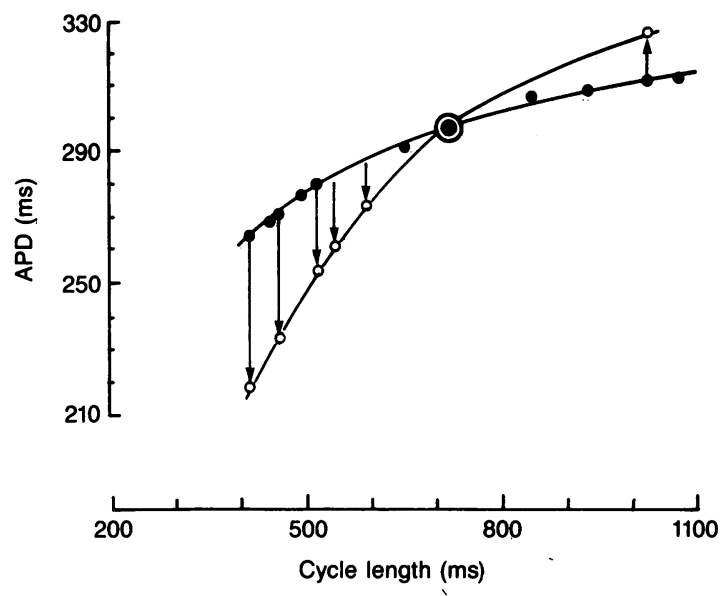

Figure 7. Comparison of APD after a single cycle-length alteration (๑, electrical restitution curve) with steady-state APD (o) at identical cycle lengths (arrows indicate direction and amount of slow adaptation). The steady-state cycle length during which single cycle length changes were introduced was $750 \mathrm{~ms}$ (the cycle length at which both curves intersect). The electrical restitution curve was truncated at $400 \mathrm{~ms}$ (the shortest steady-state cycle length in this patient) and therefore does not show the early supernormal phase. All recordings were from a single right ventricular site (patient 8). 
Electrical restitution. The time course with which APD recovers from the most premature response to its maximum at very long cycle lengths is considered to reflect time-dependent recovery from membrane activation and inactivation processes that led to repolarization of the previous response (25-28). In isolated ventricular preparations of guinea-pig (26), kitten (28), and pig (9), and in Purkinje fibers of all species studied to date $(6,9,11,27,29)$, recovery of these membrane gating variables proceeds with a smooth exponential time course. In contrast to these species and fiber types, we found the electrical restitution curve of human ventricular myocardium to be biphasic with an early peak at $\sim 50 \mathrm{~ms}$ after repolarization of the previous response. This different type of electrical restitution has been found in rabbit (8), dog $(3,4,11,30)$, sheep (31), and adult cat (25) ventricular muscle, all of which have supernormal (2) premature action potentials and an early peak of APD at electrical diastolic intervals of 50-100 ms.

The mechanism responsible for the supernormal phase of electrical restitution is not fully understood. Greenspan et al. (32) and Bass (25) demonstrated an inverse relation between action potential plateau duration and contractile force at short cycle lengths. These authors suggested that the low activator calcium concentration available for a weaker premature beat may result in increased slow inward calcium current (as a negative feedback to help restore intracellular calcium) which augments the action potential plateau duration and amplitude and, to a lesser degree, total APD. This hypothesis is supported by the observations that calcium blockers suppress supernormal action potentials and the early peak of electrical restitution (30).

In one earlier study of human ventricular APD, supernormal action potentials and a biphasic time course of electrical restitution was not observed (33). In that study, however, the time course of electrical restitution was assessed using rather large extrastimulus increments of $50 \mathrm{~ms}$. One is likely to miss the early peak of electrical restitution which occurs within a relatively narrow range of intervals. Also, patients in this earlier study had significant coronary artery disease and were treated with beta blockers at the time of study; both factors might influence the time course of electrical restitution.

Adaptation to step changes in cycle length. Beat-to-beat analysis of human APD during the entire period of steadystate adaptation, performed in this study, confirms and extends previous observations from studies on refractoriness (17, $18,34-36)$. APD adapted to a sudden decrease in cycle length in two phases: APD shortened abruptly at the first beat of the faster rate but required several hundred beats to reach final steady state. The transition from the fast to the slow phase of APD shortening was characterized by a variable pattern of APD alternans. While the first APD was always shorter than the preceding steady-state APD, subsequent responses showed either continued shortening, alternans, or no change at all for several beats. These irregular alternations in APD after sudden rate change are in close agreement with refractory period measurements of canine (35) and human (36) ventricular muscle under similar conditions, but contrast strikingly with the regular (one-to-one) oscillations seen in human His-Purkinje refractoriness after sudden rate acceleration $(18,34)$.

Action potential recordings from isolated tissue (5) and refractory period measurements in the human heart (34) have shown that APD in Purkinje fibers is predominantly a func- tion of the preceding diastolic interval, with little influence exerted by the preceding steady-state cycle length. In contrast, as shown in this study, APD of ventricular myocardium is strongly governed by the cumulative effect of the prior cycle length, in addition to its sensitivity to short-term cycle length changes.

Divergence between the symmetrical oscillations in Purkinje fiber refractoriness $(18,34)$ and more irregular alternations in ventricular refractoriness $(35,36)$ after sudden step decreases in cycle length may be explained not only by a different degree of cumulative cycle length effects but also by differences between electrical restitution curves of ventricular muscle and Purkinje fibers. As shown, the electrical restitution curve of human ventricular myocardium is biphasic with a steep rising phase and a subsequent descending limb at cycle lengths shorter than steady state. During the first few beats after a step change in cycle length, APD may shorten or lengthen, depending on whether the action potential lies on the rising or falling limb of the electrical restitution curve, or remain essentially unaltered when approaching either the peak or valley of early electrical restitution. The alternations dampen with time as the cumulative component to APD shortening gains more influence and the reciprocal lengthening of electrical diastole moves the action potential onto the flat part of the electrical restitution curve. The monophasically rising electrical restitution curve and lack of cumulative cycle length effects in Purkinje fibers would then explain the more regular oscillations of His-Purkinje refractoriness following sudden rate acceleration $(18,35)$. Calcium blockers not only suppress the supernormal phase of electrical restitution in ventricular myocardium (30) but also APD alternans after sudden rate acceleration (37), a finding that further corroborates the interpretation given above.

APD alternans has not commonly been observed in vitro. This may be due to the practice that isolated tissue preparations often are driven at rates much lower than physiological for the species under investigation (2). As indicated by the electrical restitution curve, recovery of APD between successive responses approaches a plateau at intervals of $1 \mathrm{~s}$. Consequently, changes in pacing rate not exceeding $1 \mathrm{~Hz}$ do not curtail electrical restitution between consecutive beats and cannot be expected to produce the alterations in APD that are associated with incomplete membrane recovery.

Cycle length dependence in the steady state. In the steady state, APD had a close linear correlation to cycle lengths ranging from 350 to $800 \mathrm{~ms}$ and at very long cycle lengths (above 800-1,000 ms) deviated from the linear relationship to approach a maximum plateau value. A linear relationship between APD and cycle length with flattening above cycle lengths of $1 \mathrm{~s}$ has been demonstrated previously in vitro for frog (38), canine $(10,11)$, sheep (30), cat (28) and guinea-pig ventricular muscle $(12,39)$, and canine Purkinje fibers (40). Also consistent with these previous in vitro findings, changes in the steady-state APD of human myocardium in situ were due to a change in phase 2 duration without alteration of phase 3 slope (Fig. 7).

Some species, in particular the rabbit, show "paradoxical" shortening of steady-state APD at cycle lengths longer than $2 \mathrm{~s}$ $(8,41)$. We do not know how the human APD responds to extreme bradycardia as escape beats prevented us to study the effects of cycle lengths $>1,200 \mathrm{~ms}$. Towards the low end of steady-state cycle lengths, we were able to extend our examina- 
tion to cycle lengths as short as $\mathbf{3 5 0}$ ms but chose not to pace the hearts faster than that. Recordings obtained during induced ventricular tachycardia or ventricular fibrillation in patients with clinical tachyarrhythmias, however, demonstrated that significant further shortening of APD is possible (unpublished observation). At such high heart rates, however, effects of ischemia on APD cannot be excluded.

Divergence between steady-state and non-steady-state $A P D$. The amount by which the steady-state APD differs from the APD of single premature or postmature responses at identical cycle lengths distinguishes the slow component from the fast component. The contribution of the slow component becomes increasingly important as the magnitude of the cyclelength change increases (Fig. 7); the pacing time, however, required to attain the new steady state does not necessarily increase (Table I). This suggests that even smaller step changes in paced cycle length should be maintained for similarly long times to ensure steady state.

Conclusions and clinical implications. Our results have several important clinical implications. First, because APD and refractory period are closely related, the slow time course of rate adaptation of APD should be considered during programmed electrical stimulation. Many laboratories use only eight basic stimuli before introduction of extrastimuli, and then a variable pause before beginning the next basic drive train. Our study indicates that such procedure does not guarantee steady state. This is a potential cause of variability in clinical electrophysiologic studies.

It is difficult to recommend a definitive, and clinically practical, standardized pacing protocol for electrophysiologic studies. Ideally, extrastimulation should be delivered under identical steady-state conditions to allow for comparability within the same and different individuals. This, however, does not appear clinically practical because prolonged steady-state pacing at cycle lengths of, e.g., $400 \mathrm{~ms}$ might not be safe in patients with severe coronary artery disease or cardiomyopathy. Equally important, and practical in the clinical setting, would be to keep pauses between successive drive trains at a standardized minimum, so as to minimize the loss of steadystate condition from one drive train to the next.

Secondly, the cumulative and persistent shortening of APD in response to a train of shorter cycle lengths may help explain tachycardia acceleration during electrophysiologic studies and repetitive paroxysms of tachyarrhythmias. After the onset of tachycardia, progressive shortening of APD with concomitant decrease of the refractory period might shorten the reentry circuit time. Persistence of APD shortening after termination of a tachyarrhythmia may permit earlier reexcitation than is possible at a similar cycle length before initiation of the tachyarrhythmia, and thereby enhance vulnerability to tachyarrhythmias.

Thirdly, our findings that ventricular myocardium has a biphasic electrical restitution curve and an APD alternans which contrast with those in Purkinje fibers may pertain to mechanisms of tachyarrhythmia induction by programmed electrical stimulation. Divergence in the response to abrupt cycle length changes between ventricular muscle and Purkinje fibers enhances dispersion of refractoriness between these two tissue types, a situation which long has been suspected to facilitate reentrant tachycardias $(42,43)$.

Finally, the results of our study challenge the validity of the Bazett rate-correction algorithm of the QT interval. The Bazett formula assumes a square-root relation between heart rate and QT interval, irrespective of whether repolarization time of the myocardium has been allowed to attain steady state or not (44). Our study shows that the relationship between cycle length and APD (and by inference that of QT interval) after transient rate changes differs from that in the steady state. For instantaneous cycle length alterations (such as in premature beats) the QT interval should be governed by the electrical restitution curve. In the steady state, APD is linearly correlated to cycle length, increasing an average $23 \mathrm{~ms}$ with every $100-\mathrm{ms}$ cycle length increment. It should be of interest to determine whether, under true steady-state conditions, the QT interval also follows this much simpler algorithm of cycle length dependence.

\section{Acknowledgments}

Dr. Franz is the recipient of a FIRST award (1 R29 HL-40482-01) from the National Heart, Lung and Blood Institute.

\section{References}

1. Carmeliet, R. 1977. Repolarisation and frequency in cardiac cells. J. Physiol. (Paris). 73:903-923.

2. Boyett, M. R., and B. R. Jewell. 1980. Analysis of the effects of changes in rate and rhythm upon the electrical activity in the heart. Prog. Biophys. Mol. Biol. 36:1-52.

3. Edmands, R. E., K. Greenspan, and C. Fisch. 1966. Effect of cycle length alteration upon the configuration of the canine ventricular action potential. Circ. Res. 19:602-610.

4. Greenspan, K., R. E. Edmands, and C. Fisch. 1967. Effects of cycle-length alteration on canine cardiac action potentials. Am. J. Physiol. 212:1416-1420.

5. Elharrar, V., H. Atarashi, and B. Surawicz. 1984. Cycle lengthdependent action potential duration in canine cardiac Purkinje fibers. Am. J. Physiol. 247:H936-H945.

6. Colatsky, T. J., and P. M. Hogan. 1980. Effects of external calcium, calcium channel-blocking agents, and stimulation frequency on cycle length-dependent changes in canine cardiac action potential duration. Circ. Res. 46:543-552.

7. Arnold, L., J. Page, D. Attwell, M. Cannell, and D. A. Eisner. 1982. The dependence on heart rate of the human ventricular action potential duration. Cardiovasc. Res. 16:547-551.

8. Gibbs, C. L., and E. A. Johnson. 1961. Effect of changes in frequency of stimulation upon rabbit ventricular action potential. Circ. Res. 9:165-170.

9. Gettes, L. S., N. Morehouse, and B. Surawicz. 1972. Effect of premature depolarization on the duration of action potentials in Purkinje and ventricular fibers of the moderator band of the pig heart: role of proximity and the duration of the preceding action potential. Circ. Res. 30:55-66.

10. Hoffman, B. F., and E. E. Suckling. 1954. Effect of heart rate on cardiac membrane potentials and the unipolar electrogram. Am. J. Physiol. 179:123-130.

11. Miller, J. P., A. G. Wallace, and M. D. Feezor. 1971. A quantitative comparison of the relation between the shape of the action potential and the pattern of stimulation in canine ventricular muscle and Purkinje fibers. J. Mol. Cell. Cardiol. 2:3-19.

12. Savigny, L., S. Hohnloser, and H. Antoni. 1981. Effects of changes in frequency on guinea pig ventricular APD and on QT interval under different experimental conditions. Basic Res. Cardiol. 76:276-288.

13. Attwell, D., I. Cohen, and D. A. Eisner. 1981. The effects of heart rate on the action potential of guinea-pig and human ventricular muscle. J. Physiol. (Lond.). 313:439-461.

14. Watanabe, T., P. M. Rautaharju, and T. F. McDonald. 1985. 
Ventricular action potentials, ventricular extracellular potentials, and the ECG of guinea-pig. Circ. Res. 57:362-373.

15. Abildskov, J. A. 1975. The sequence of normal recovery of excitability in the dog heart. Circulation. 52:442-446.

16. Franz, M. R., K. Bargheer, W. Rafflenbeul, A. Haverich, and P. R. Lichtlen. 1987. Monophasic action potential mapping in human subjects with normal electrocardiograms: direct evidence for the genesis of the T-wave. Circulation. 75:379-386.

17. Wiener, I., S. Kunkes, D. Rubin, J. Kupersmith, M. Packer, R. Pitchon, and P. Schweitzer. 1981. Effects of sudden change in cycle length on human atrial, atrioventricular nodal and ventricular refractory periods. Circulation. 64:245-248.

18. Tchou, P. J., M. H. Lehmann, J. Dongas, R. Mahmud, S. T. Denker, and M. Akhtar. 1986. Effect of sudden rate acceleration on the human His-Purkinje system: adaptation of refractoriness in a dampened oscillatory pattern. Circulation. 73:920-929.

19. Akhtar, M., S. T. Denker, M. H. Lehmann, and R. Mahmud. 1985. Effects of sudden cycle length alteration on refractoriness of human His-Purkinje system and ventricular myocardium. In Cardiac Electrophysiology and Arrhythmias. D. P. Zipes and J. Jalife, editors. Grune \& Stratton, Inc., Orlando, FL. p 399-407.

20. Olsson, S. B. 1972. Right ventricular monophasic action potentials during regular rhythm. A heart catheterization study in man. Acta Med. Scand. 191:145-157.

21. Olsson, S. B. 1971. Monophasic action potentials of right heart. Suction electrode method in clinical investigation. Elanders Boktryckeri Aktiebolag, Göteborg, Sweden. 10.

22. Franz, M. R. 1983. Long-term recording of monophasic action potentials from human endocardium. Am. J. Cardiol. 51:1629-1634.

23. Franz, M. R., D. Burkhoff, H. Spurgeon, M. L. Weisfeldt, and E. G. Lakatta. 1986. In vitro validation of new cardiac catheter technique for recording monophasic action potentials. Eur. Heart J. 7:34-41.

24. Franz, M. R., S. O. Gottlieb, and J. A. Brinker. 1983. Renografin-76 but not Iopamidol produces marked local prolongation of canine monophasic action potential duration. Circulation. 68(Suppl.):III-350. (Abstr.)

25. Bass, B. G. 1975. Restitution of the action potential in cat papillary muscle. Am. J. Physiol. 228:1717-1724.

26. Gettes, L. S., and H. Reuter. 1974. Slow recovery from inactivation of inward currents in mammalian myocardial fibres. J. Physiol. (Lond.). 240:703-724.

27. Hauswirth, O., D. Noble, and R. W. Tsien. 1972. The dependence of plateau currents in cardiac Purkinje fibres on the interval between action potentials. J. Physiol. (Lond.). 222:27-49.

28. Boyett, M. R., and B. R. Jewell. 1978. A study of the factors responsible for rate-dependent shortening of the action potential in mammalian ventricular muscle. J. Physiol. (Lond.). 285:359-380.

29. Vick, R. L. 1971. Action potential duration in canine Purkinje tissue: effects of preceding excitation. J. Electrocardiol. 4:105-115.

30. Iinuma, H., and K. Kato. 1979. Mechanism of augmented premature responses in canine ventricular muscle. Circ. Res. 44:624629.

31. Noble, D., and I. Cohen. 1978. The interpretation of the T wave of the electrocardiogram. Cardiovasc. Res. 12:13-27.

32. Greenspan, K., and R. E. Edmands. 1967. The relation of contractile enhancement to action potential change in canine myocardium. Circ. Res. 20:311-320.

33. Seed, W. A., M. I. M. Noble, P. Oldershaw, R. B. Wanless, A. J. Drake-Holland, D. Redwood, S. Pugh, and C. Mills. 1987. Relation of human action potential duration to the interval between beats: implications for the validity of rate corrected QT interval (QTc). Br. Heart J. 57:32-37.

34. Denker, S., M. Shenasa, C. J. Gilbert, and M. Akhtar. 1983. Effects of abrupt changes in cycle length on refractoriness of the HisPurkinje system in man. Circulation. 67:60-68.

35. Janse, M. J., A. B. M. van der Steen, R. Th. van Dam, and D. Durrer. 1969. Refractory period of the dog's ventricular myocardium following sudden changes in frequency. Circ. Res. 24(Suppl. 1):251262.

36. Marchlinski, F. E. 1987. Characterization of oscillations in ventricular refractoriness in man after an abrupt increment in heart rate. Circulation. 75:550-556.

37. Hirata, Y., I. Kodama, N. Wamura, T. Shimizu, J. Toyama, and K. Yamada. 1979. Effects of verapamil on canine Purkinje fibres and ventricular muscle fibres with particular reference to the alternation of action potential duration after a sudden increase in driving rate. Cardiovasc. Res. 13:1-8.

38. Carmeliet, E. 1958. Modification de la durée du potentiel d'action cardiaque sous l'influence des excitants. J. Physiol. (Paris). 50:204-207.

39. Ravens, U. 1983. Aktionspotentialform, Kontraktionskraft und Frequenz: Untersuchungen an Warmblüterherzen. GeorgThieme-Verlag, Stuttgart.

40. Moore, E. N., J. B. Preston, and G. K. Moe. 1965. Durations of transmembrane action potentials and functional refractory periods of canine false tendon and ventricular myocardium. Comparisons in single fibers. Circ. Res. 27:259-273.

41. Ino, T., H. S. Karagueuzian, K. Hong, M. Meesman, W. Mandel, T. Peter. 1987. The relation of contact monophasic action potentials to underlying myocardial cell population. A microelectrode validation study. PACE (Pacing Clin. Electrophysiol.). 10(II):987. (Abstr.)

42. Kuo, C. S., K. Munakata, C. P. Reddy, and B. Surawicz. 1983. Characteristics and possible mechanism of ventricular arrhythmia dependent on the dispersion of action potential durations. Circulation. 67:1356-1367.

43. Denker, S., M. Lehmann, R. Mahmud, C. Gilbert, and M. Akhtar. 1984. Facilitation of ventricular tachycardia induction with abrupt changes in ventricular cycle length. Am. J. Cardiol. 53:508515.

44. Bazett, H. C. 1920. An analysis of the time relations of electrocardiograms. Heart. 7:353-370. 